\title{
Phase Separation in Multicomponent Aqueous-Protein Solutions
}

\author{
Canwen Liu, Aleksey Lomakin, George M. Thurston, ${ }^{\dagger}$ Douglas Hayden, ${ }^{\dagger}$ Ajay Pande, ${ }^{\ddagger}$ \\ Jayanti Pande, Olutayo Ogun, Neer Asherie, and George B. Benedek* \\ Department of Physics and Center for Materials Sciences and Engineering, M.I.T., \\ Cambridge, Massachusetts 02139
}

Received: July 25, 1994; In Final Form: October 14, $1994^{\otimes}$

\begin{abstract}
We present measurements of the phase-separation temperature $\left(T_{\mathrm{ph}}(\phi, \alpha)\right)$ as a function of overall protein volume fraction $(\phi)$ and protein composition $(\alpha)$ for ternary aqueous $(W)$ solutions of calf $\gamma_{\mathrm{IIa}}(A)$ and $\gamma_{\mathrm{III}}$ $(B)$ crystallins. Additionally, we have determined the binodal curve describing coexisting points $\left(\phi^{I}, \alpha^{\mathrm{I}}\right)$ and $\left(\phi^{\mathrm{I}}, \alpha^{\mathrm{II}}\right)$ in the phase diagram at $20^{\circ} \mathrm{C}$. We propose a mean-field form of the ternary Gibbs free energy $G(\phi, \alpha, T)$ which contains three interaction energy parameters: $E_{n e t}(A, W), E_{n e t}(B, W)$, and $E_{n e t}(A, B)$, which determine the magnitude of the quadratic $\left(\phi^{2}\right)$ mixing energy contribution to $G$. Using a lattice model it is possible to express each of these interaction parameters in terms of the mean individual protein-water, proteinprotein and water-water bond energies. In the limit where the two proteins are not too dissimilar, as applies in our system, we find quite generally that the ternary solution can be regarded as a binary solution with an interaction energy dependent upon the initial composition $(\alpha)$ of the solution. We have used this finding to predict the entire coexistence surface $T_{p h}(\phi, \alpha)$ and the positions of coexisting points along the binodal curve. The interaction energy parameters were determined and we show that, within experimental error, this theory accurately describes the full range of our experimental results.
\end{abstract}

\section{Introduction}

We have previously published ${ }^{1}$ measurements of the coexistence curves which describe the separation of binary aqueousprotein solutions into coexisting protein-rich and protein-poor phases. Such phase separation phenomena have attracted our attention because a wide class of human diseases including cataract in eye lens, ${ }^{2}$ sickle cell anemia ${ }^{3}$ cryoimmunoglobulinemia, and a broad group of amyloidoses ${ }^{4}$ including amyloid plaque formation in Alzheimer's disease ${ }^{5}$ involve the condensation of protein into such coexisting protein-rich and proteinpoor phases. Our previous studies focused on aqueous solutions, each of which contained a single highly purified species of protein. The individual proteins studied were members of the calf lens $\gamma$-crystallin family, namely, $\gamma_{\mathrm{II}}$ and $\gamma_{\mathrm{Imb}}$, which exhibit low critical temperatures $\left(T_{\mathrm{c}} \approx 5^{\circ} \mathrm{C}\right)$, and $\gamma_{\mathrm{maa}}$ and $\gamma_{\mathrm{IVa}}$, which have high critical temperatures $\left(T_{\mathrm{C}} \approx 38^{\circ} \mathrm{C}\right)$. The amino acid sequences for the calf, rat, and human $\gamma$-crystallins are very similar. An examination of sequence homologies indicates that these proteins can be arranged into six orthologous groups $\gamma_{\mathrm{A}}$ $\gamma_{\mathrm{F}}$. In the case of the calf lens, the crystallins we designate as $\gamma_{\mathrm{II}}, \gamma_{\mathrm{III}}, \gamma_{\mathrm{IIb}}$, and $\gamma_{\mathrm{IVa}}$ correspond in the current gene sequence designation $^{6}$ to $\gamma_{\mathrm{B}}, \gamma_{\mathrm{C}}, \gamma_{\mathrm{D}}$, and $\gamma_{\mathrm{E}}$, respectively. The minor constituent, $\gamma_{\mathrm{IVb}}$, of the $\gamma_{\mathrm{IV}}$ fraction remains to be clearly identified as being either $\gamma_{\mathrm{A}}$ or $\gamma_{\mathrm{F}}$. In the case of the living cell, of course, the phase separation phenomenon takes place in aqueous solutions containing multiple protein species. Furthermore, in the search for pharmaceutical agents which can alter the location of the coexistence curve, we conduct in vitro assays using at least ternary aqueous-protein solutions. It is therefore quite important to understand both experimentally and theoretically the quantitative factors which govern phase separation in multicomponent aqueous-protein solutions. To this end,

\footnotetext{
$\dagger$ Oculon Corporation, 26 Landsdowne Street, Cambridge, MA 02139.

¥ Boston Biomedical Research Institute, 20 Staniford Street, Boston, MA 02114.

* To whom correspondence should be addressed.

${ }^{\star}$ Abstract published in Advance ACS Abstracts, December 1, 1994.
}

we present here the results of experimental and theoretical studies of a ternary solution constituted of the proteins $\gamma_{\mathrm{IIa}}$ (designated as protein $\mathrm{A}$ ), a "high- $T_{C}$ " protein, and $\gamma_{\mathrm{mb}}$ (designated as protein B), a "low- $T_{C}$ " protein, in aqueous solution. The solution pH was kept constant at 7.1 by a 100 $\mathrm{mM}$ phosphate buffer and the solution ionic strength was 240 $\mathrm{mM}$. Both these proteins have the same effective volume $\Omega_{P}$ as their molecular weights are essentially the same and equal to $21 \mathrm{kDa}$.

To facilitate the subsequent presentation it will be useful to introduce the fundamental quantities which we shall use to describe these solutions. Let $N_{A}, N_{B}$, and $N_{W}$ be the total number of proteins $A$ and $B$ and water molecules in the solution. The effective volumes $\Omega_{P}$ and $\Omega_{W}$ of protein and water molecules are chosen to subsume the effect of the ions so that the total volume $V$ is given by $V=N_{A} \Omega_{P}+N_{B} \Omega_{P}+N_{W} \Omega_{W}$. In this work two sets of state variables naturally suggest themselves to describe the system. The first set $\left(T, \phi_{A}, \phi_{B}\right)$ are the temperature, the volume fractions of proteins $A$ and $B$, and the volume fraction of water:

$$
\phi_{A}=N_{A} \Omega_{P} / V ; \quad \phi_{B}=N_{B} \Omega_{P} / V ; \quad \phi_{W}=N_{W} \Omega_{W} / V
$$

where

$$
\phi_{A}+\phi_{B}+\phi_{W}=1
$$

The second natural set of state variables is $(T, \phi, \alpha)$ where $T$ is the temperature, $\phi$ is the total protein volume fraction, and $\alpha$ is the number fraction of protein $A$, which we shall designate as "the composition". According to these definitions,

$$
\phi=\left(N_{A}+N_{B}\right) \Omega_{P} / V ; \quad \alpha=N_{A} /\left(N_{A}+N_{B}\right)
$$

Clearly the two sets of state variables are connected by the conditions

$$
\phi_{A}=\alpha \phi ; \phi_{B}=(1-\alpha) \phi ; \phi_{W}=(1-\phi)
$$


When the system separates into coexisting phases we will use the symbols I and II to designate respectively small $\phi$ and large $\phi$ phases. With these definitions in mind we can now summarize the experimental findings to be reported.

Let us define $T_{p h}(\phi, \alpha)$ as the temperature at which phase separation occurs for fixed values of $\phi$ and $\alpha$. The surface $T_{p h}(\phi, \alpha)$ can be designated as the "coexistence surface". In our experiments we have determined the curves, $\left(T_{p h}(\phi)\right)_{\alpha}$, of intersection of planes of fixed $\alpha$ with the coexistence surface. When $\alpha=0$ and $\alpha=1$ these intersections correspond to the coexistence curves of pure $B$ and pure $A$ solutions, respectively. We have also determined the curves, $\left(T_{p h}(\alpha)\right)_{\phi}$, of intersection of planes of fixed $\phi$ with the coexistence surface. Finally, we have determined at fixed temperature $T$ the coexisting values of protein volume fraction $\phi$ and composition $\alpha$ : i.e., $\left(\phi^{I}, \alpha^{I}\right)$ and $\left(\phi^{I I}, \alpha^{I I}\right)$. Each such pair of points lies at distinct locations on the curved line of intersection of the constant-temperature plane and the coexistence surface. This curved intersection is called the "binodal curve". The line joining $\left(\phi^{I}, \alpha^{I}\right)$ and $\left(\phi^{I l}, \alpha^{I I}\right)$ is called the "tie line". We have determined several such tie lines at fixed temperature by using a variety of starting compositions of our test solutions.

We also present a theoretical analysis of the experimental findings. According to this analysis is follows quite generally that, if the interaction energy between unlike proteins is close to the mean of the like-like protein interaction energies, then in first approximation the $\left(T_{p h}(\phi)\right)_{\alpha}$ sections on the coexistence surface for each composition $\alpha$ have a very simple property: each such section is simply a vertical displacement of the same curve whose shape is independent of $\alpha$. This prediction is in fact found to apply for our experimental results for $\gamma_{\mathrm{ma}}$ and $\gamma_{\mathrm{IIb}}$ solutions. ${ }^{5}$ To interpret our results, in quantitative detail, we have constructed a mean-field form of the Gibbs free energy appropriate for such ternary solutions. This free energy contains three interaction energy parameters. The physical meaning of each parameter is identified by using a lattice model for the ternary solution. The magnitudes and temperature dependences of two of these parameters are fixed by the location of the phase boundaries of the pure $A$ and pure $B$ solutions. ${ }^{7}$ By fixing the magnitude of the single remaining parameter, it is possible to satisfactorily predict all our experimental findings on the coexistence surface and the tie lines of the binodal curve describing this ternary solution.

\section{Materials and Methods}

Protein Isolation and Purification. The $\gamma$-crystallins used in our study were isolated from 1-6-week-old calf lenses, obtained by overnight express from Antech (Tyler, TX). The monomeric $\gamma$-crystallin fraction was isolated from the soluble protein fraction by size-exclusion chromatography on Sephadex G-75, as described earlier. ${ }^{8}$ Native $\gamma$-crystallin so obtained was further fractionated into $\gamma_{\mathrm{I}}, \gamma_{\mathrm{S}}, \gamma_{\mathrm{II}}, \gamma_{\mathrm{II}}$, and $\gamma_{\mathrm{IV}}$ by cationexchange chromatography on sulfopropyl Sephadex C-50, essentially according to Björk ${ }^{9}$ and Thomson et al. ${ }^{8}$ Anionexchange chromatography on diethylaminoethyl (DEAE)Sephadex was used to fractionate $\gamma_{\mathrm{III}}$ into $\gamma_{\mathrm{IIa}}$ and $\gamma_{\mathrm{mb}}$ as described in Broide et al. ${ }^{1}$ Purity of the $\gamma_{\mathrm{III}}$ and $\gamma_{\mathrm{ILb}}$ fractions was at least $95 \%$, based on cation-exchange high-performance liquid chromatography (HPLC) according to Siezen et al. ${ }^{10}$ and isoelectric focusing. ${ }^{11}$ The purified crystallin fractions were dialyzed exhaustively into $100 \mathrm{mM}$ sodium phosphate buffer (ionic strength $240 \mathrm{mM}, \mathrm{pH} 7.1$ ), which contained sodium azide (3 mM).

Experimental Methods. Pure solutions of $\gamma_{\mathrm{IIa}}$ and $\gamma_{\mathrm{mb}}$ crystallins were prepared separately according to direct ultra- filtration and/or a quench method as detailed in Broide et al. ${ }^{1}$ Precautions were taken to obtain single-phase solutions. Two issues of concern were crystal formation and aggregation. We followed Broide et al. ${ }^{1}$ and Berland et al..$^{12}$ to obtain crystalfree solutions. We observed, during the entire experimental period, two phenomena: reversible two-phase formation and a sharp boundary separating the macroscopic protein-poor and protein-rich phases. These results suggested that aggregate formation was negligible. The absence of aggregation was confirmed by HPLC runs. The concentrations (in $\mathrm{mg} / \mathrm{mL}$ ) for pure protein solutions were determined by measuring the UV absorbance at $280 \mathrm{~nm}$ using the extinction coefficients ${ }^{1}$ $E_{280}^{0.1 \%}, 1 \mathrm{~cm}\left(\gamma_{\mathrm{IIa}}\right)=2.33$ for the pure $\gamma_{\mathrm{III}}$ crystallin and $E_{280}^{0.1 \%, 1 \mathrm{~cm}}\left(\gamma_{\mathrm{Imb}}\right)=2.11$ for the pure $\gamma_{\mathrm{Imb}}$ crystallin. A ternary mixture was made by mixing pure $\gamma_{\mathrm{IIa}}$ solution and pure $\gamma_{\mathrm{Imb}}$ solution at known concentrations according to a desired composition and was stirred to ensure thorough mixing. The total protein concentration of the mixture was determined in the following way. A small amount of the mixture $(20 \mu \mathrm{L})$ was taken and diluted to about $15 \mathrm{mg} / \mathrm{mL}$, and an HPLC experiment was run to determine its composition $\alpha$. The extinction coefficient for the mixture at composition $\alpha$ was calculated from the weight percentages of each of the two proteins present in the mixture: $E_{280}^{0.1 \%, 1 \mathrm{~cm}}(\alpha)=$ $\alpha E_{280}^{0.1 \%, 1 \mathrm{~cm}}\left(\gamma_{\mathrm{ma}}\right)+(1-\alpha) E_{280}^{0.1 \%, 1 \mathrm{~cm}}\left(\gamma_{\mathrm{mbb}}\right)$. The total UV absorbance at $280 \mathrm{~nm}$ of the mixture was measured and the total protein concentration (in $\mathrm{mg} / \mathrm{mL}$ ) was determined through $E_{280}^{0.1 \%, 1 \mathrm{~cm}}(\alpha)$. We usually use the dimensionless volume fraction $\phi$ of the protein instead of its concentration $C$ in $\mathrm{mg} / \mathrm{mL}$. The value of $\phi$ is calculated from the measurement of $C$ by using the expression $\phi=\bar{\nu} C$, where $\bar{\nu}$ is the partial specific volume of the protein. ${ }^{13}$ For $\gamma_{\mathrm{II}}$ crystallin, $\bar{v}=7.1 \times 10^{-4}$ $\mathrm{cm}^{3} / \mathrm{mg} ;{ }^{14}$ we assume that the specific volume is approximately the same for the other $\gamma$-crystallins.

To determine the temperature $\left(T_{p h}(\phi)\right)_{\alpha}$ for phase separation as a function of total protein volume fraction $\phi$ at fixed compositions $\alpha=0.18,0.28,0.64$, a dilute ternary mixture at desired composition $\alpha$ was prepared and subjected to further ultrafiltration to produce as concentrated a sample as possible. The composition $\alpha$ and volume fraction $\phi$ of the sample were determined by using HPLC and UV absorbance at $280 \mathrm{~nm}$. The temperature of the solution was then lowered until the phaseseparation temperature $T_{p h}$ was found by using the cloud point method. ${ }^{1}$ This sample was then diluted to produce a lower volume fraction $\phi$. For each such dilution $T_{p h}$ was determined and $\phi$ was measured by using UV absorbance. Several HPLC runs were made at different dilutions to determine the average composition $\bar{\alpha}$ for each section of the coexistence surface. The extinction coefficient of the mixture $E_{280}^{0.1 \%, 1 \mathrm{~cm}}(\bar{\alpha})$ was used in the determination of each $\phi$ for fixed $\bar{\alpha}$.

To determine the temperature $\left(T_{p h}(\alpha)\right)_{\phi}$ for phase separation as a function of composition $\alpha$ at fixed total protein volume fraction $\phi=0.04,0.09,0.14$, and 0.20 , we prepared pairs of pure $\gamma_{\mathrm{III}}$ and pure $\gamma_{\mathrm{mb}}$ samples having approximately equal volume fractions. Each pair of samples was then combined in different proportions so as to produce a series of samples within each of which the total volume fraction of protein was the same, but the relative proportion of the individual proteins was varied. The composition, $\alpha$, of the mixture was calculated for each sample from the amount of $\gamma_{\mathrm{ma}}$ present and the total amount of protein present. The cloud point method was used to determine $T_{p h}$ for each sample.

Finally, to determine the coexisting values $\left(\phi^{I}, \alpha^{I}\right)$ and $\left(\phi^{I I}, \alpha^{I I}\right)$, which determine the tie lines connecting points on the binodal curve, we induced macroscopic phase separation (two-layer 


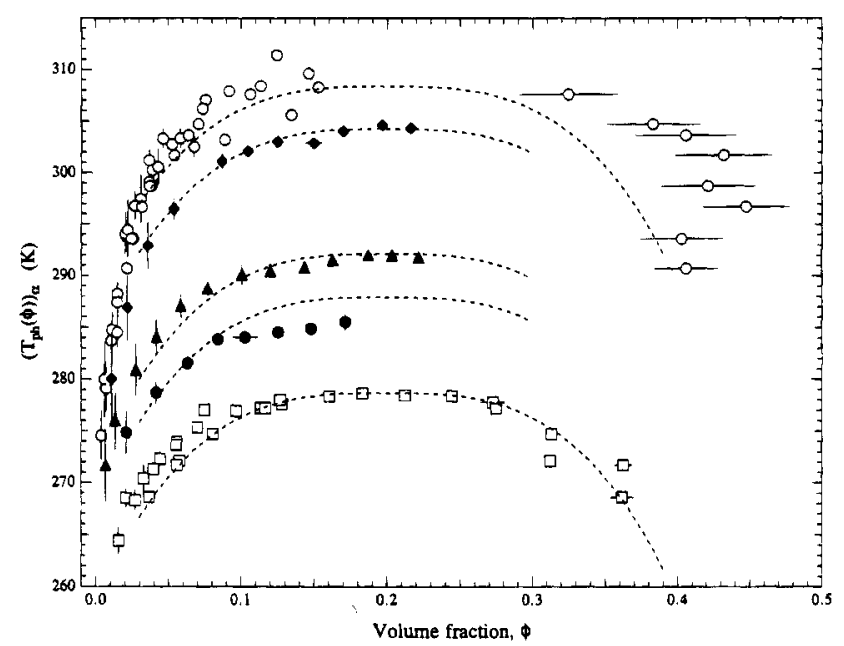

Figure 1. $\left(T_{p h}(\phi)\right)_{a}$ sections of the coexistence surface for ternary solutions of purified calf $\gamma$-crystallin $\gamma_{\mathrm{mbb}}$ and $\gamma_{\mathrm{ma}}$ at fixed compositions: $\alpha=0.0(\square) ; \alpha=0.18(\bullet) ; \alpha=0.28(\Delta) ; \alpha=0.64(\diamond) ; \alpha=$ $1.0(O)$. The dashed lines represent the theoretical predictions. The data for $\alpha=0.0\left(\gamma_{\mathrm{Imb}}\right)$ and $\alpha=1.0\left(\gamma_{\mathrm{ma}}\right)$ were taken from ref 1 . The solution conditions were at $\mathrm{pH} 7.1$ in $100 \mathrm{mM}$ phosphate buffer/ionic strength $240 \mathrm{mM}$.

formation) of the mixture by quenching to a fixed temperature which is below the phase separation temperature of the initial homogeneous sample. Several initial ternary mixtures at known total protein concentrations and compositions $\alpha^{0}=0.88,0.70$, 0.62 , and 0.50 were prepared. Each sample was placed in a small test tube (6-mm diameter) and quenched in a water bath which was stabilized at $20{ }^{\circ} \mathrm{C}$. The phase separation immediately occurred, and the dense (protein-rich) phase sedimented to the bottom of the test tube due to gravity. Centrifugation at $2000 \mathrm{~g}$ (with the centrifuge being regulated to be around the quench temperature) was used to speed up the sedimentation process. The samples were then left in the water bath until a sharp interface was formed between the two layers. We took this as an indication of equilibrium. The time for this to occur varied from two days to a week depending on the compositions of the samples; the greater the proportion of $\gamma_{\mathrm{IIa}}$ in the mixture, the longer it took for this to be achieved. After the sharp interface was attained, the upper dilute phase was withdrawn by a micropipette. The interface was carefully removed to ensure that as little as possible to the dilute phase remained. Diluted samples from both the upper dilute phase and bottom dense phase were made for the determination of the compositions (by HPLC) and total protein concentrations (by UV absorbance at $280 \mathrm{~nm}$ ) of each phase.

\section{Experimental Results}

In Figure 1 we present our measurements of the intersections $\left(T_{p h}(\phi)\right)_{\alpha}$ of the planes of constant $\alpha=0.0,0.18,0.28,0.64$, and 1.0 on the coexistence surface for the ternary $\gamma_{\mathrm{IIa}}, \gamma_{\mathrm{mb}}$, and water system. The data corresponding to $\alpha=0$ and $\alpha=$ 1 are taken from Broide et al. ${ }^{1}$ We observe that over the entire range of $\alpha$ these sections of the coexistence surface retain the same shape. Changes in the values of $\alpha$ simply shift each curve vertically.

In Figure 2 we present our measurements of the intersections $\left(T_{p h}(\alpha)\right)_{\phi}$ of the planes of constant $\phi$ on the coexistence surface for $\phi=0.04,0.09,0.14$, and 0.20 .

In Figure 3 we present our experimental data (solid symbols) on the coexisting values $\left(\phi^{I}, \alpha^{I}\right)$ and $\left(\phi^{I I}, \alpha^{I I}\right)$ found upon quenching the ternary mixture below the coexistence surface to a temperature $T=20^{\circ} \mathrm{C}$. These data are also presented in

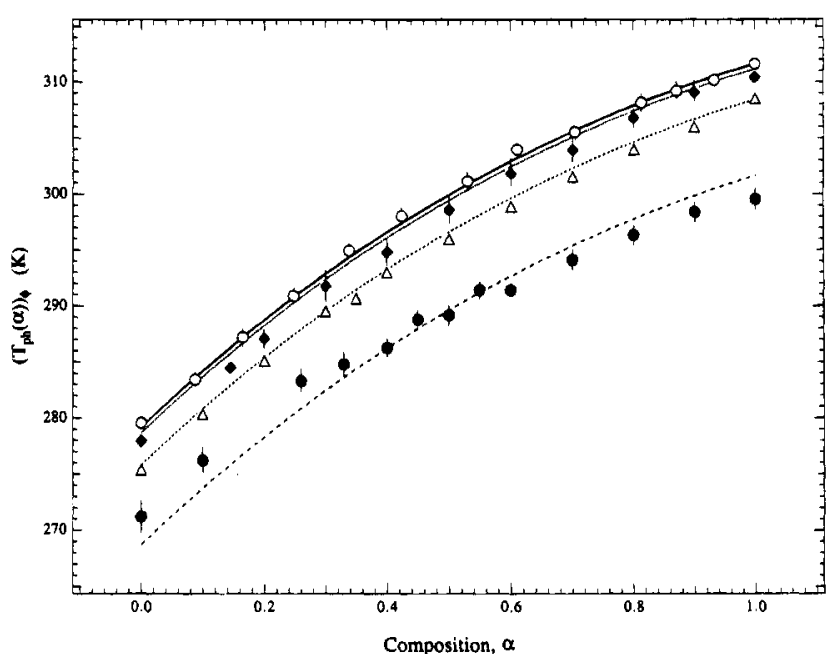

Figure 2. $\left(T_{p h}(\alpha)\right)_{\phi}$ sections of the coexistence surface as a function of composition $\alpha$ at fixed total protein volume fractions: $\phi=0.20$ $(O) ; \phi=0.14(\diamond) ; \phi=0.09(\Delta) ; \phi=0.04(\odot)$. The continuous lines shown are the theoretical predictions using the values of $t(\phi)$ and the energy parameters presented in the text.

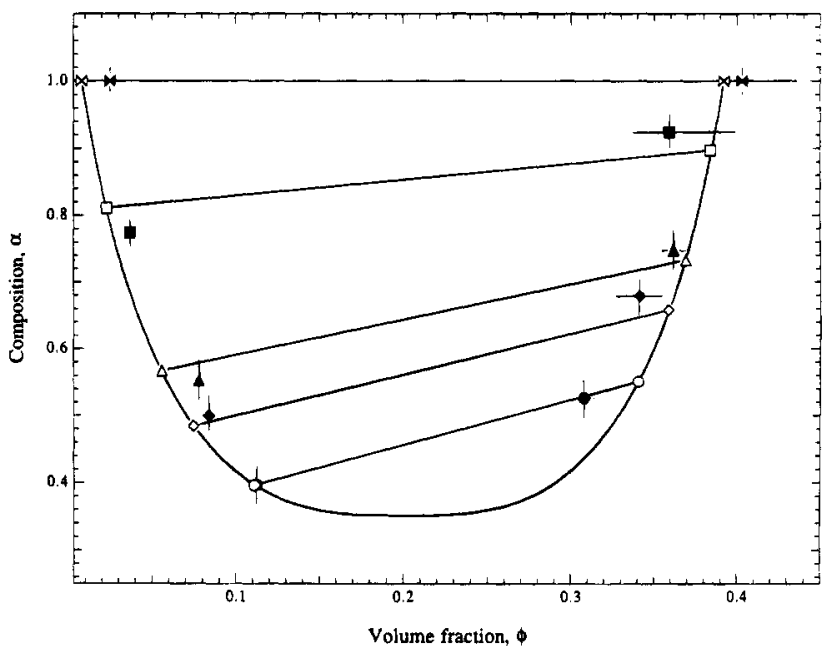

Figure 3. Coexisting values $\left(\phi^{l}, \alpha^{l}\right)$ and $\left(\phi^{I I}, \alpha^{I I}\right)$ found upon quenching the ternary mixture below the coexistence surface to a fixed temperature $T=20^{\circ} \mathrm{C}$ at several initial compositions: $\alpha^{0}=0.50$ (circles); $\alpha^{0}=$ 0.62 (diamonds); $\alpha^{0}=0.70$ (triangles); $\alpha^{0}=0.88$ (squares); $\alpha^{0}=1.0$ (bow ties). The last pair of quench data points (bow ties) for a pure $\gamma_{\mathrm{ma}}$-water binary solution was taken from ref 1 . The solid symbols are the experimental results. The solid curve and the open symbols represent the theoretically calculated binodal curves and coexisting values of $\phi$ and $\alpha$, respectively. The slopes of the straight lines connecting coexisting phases are consistent with eq 20 . It should be noted that in the $(\phi, \alpha)$ representation, starting values of $\phi^{0}$ and $\alpha^{0}$ do not generally lie on the straight line connecting $\left(\phi^{I}, \alpha^{I}\right)$ and $\left(\phi^{I I}, \alpha^{I I}\right)$.

TABLE 1: Coexisting Values $\left(\phi^{\mathrm{I}}, \alpha^{\mathrm{I}}\right)$ and $\left(\phi^{\mathrm{II}}, \alpha^{\mathrm{II}}\right)$ at $T=20$ ${ }^{\circ} \mathrm{C}$ along with the Initial Values of Total Protein Volume Fraction $\phi^{0}$ and Composition $\alpha^{0}$

\begin{tabular}{cccccc}
\hline$\phi^{\mathrm{I}}$ & $\alpha^{\mathrm{I}}$ & $\phi^{\text {II }}$ & $\alpha^{\text {II }}$ & $\phi^{0}$ & $\alpha^{0}$ \\
\hline 0.112 & 0.396 & 0.308 & 0.526 & 0.206 & 0.502 \\
0.084 & 0.499 & 0.341 & 0.679 & 0.175 & 0.624 \\
0.078 & 0.552 & 0.362 & 0.747 & 0.168 & 0.698 \\
0.037 & 0.774 & 0.336 & 0.924 & 0.098 & 0.881
\end{tabular}

Table 1, along with the initial values of total protein volume fraction $\phi^{0}$ and composition $\alpha^{0}$. The solid straight lines, the solid curve, and the open symbols in Figure 3 represent the tie lines, the binodal curve, and the coexisting values of $\phi$ and $\alpha$, respectively, calculated from the theory which is to be discussed in the next section.

We have also conducted preliminary investigations of a few 
sections of the coexistence surface for aqueous solutions of $\gamma_{\mathrm{IVa}}$ and $\gamma_{\mathrm{IIb}}$ and $\gamma_{\mathrm{IV}}$ and $\gamma_{\mathrm{II}}$ under the same solution conditions as those described here. These systems exhibit behavior qualitatively similar to the case of $\gamma_{\mathrm{III}}$ and $\gamma_{\mathrm{IIb}}$ reported above.

\section{Theory}

All the equilibrium thermodynamic properties, including the equation of state, and the location of the phase boundaries for liquid-liquid phase separation can be deduced from the structure of the Gibbs free energy at fixed pressure $G\left(N_{A}, N_{B}, N_{W}, T\right)$. In view of the extensivity of $G$, it can be expressed in terms of an intensive function: the "normalized Gibbs free energy" $g(\phi, \alpha, T)$, which is related to $G$ by

$$
g(\phi, \alpha, T)=\left(\Omega_{P} / V\right) G\left(N_{A}, N_{B}, N_{W}, T\right)
$$

Here the intensive variables $(\phi, \alpha)$ defined in eq (2) are used in place of the extensive variables $N_{A}, N_{B}$, and $V$, and $N_{W}$ is eliminated in favor of $N_{A}$ and $N_{B}$ by using the conservation of volume condition as expressed in eqs $1 \mathrm{a}$ and $1 \mathrm{~b}$. In previous work ${ }^{7}$ we have shown that in the case of a binary mixture of pure protein $A$ in aqueous solution, all experimental data could be described by using the following form of the normalized Gibbs free energy $g_{A}(\phi, T)$ :

$$
g_{A}(\phi, T)=g_{A}^{0}+k T f(\phi)-\sigma E_{n e t}(A, W) \phi^{2}
$$

Here $g_{\mathrm{A}}^{0}$ represents the "standard part" of the normalized Gibbs free energy and contains terms at most linear on $\phi$, viz.,

$$
g_{A}^{0}=\gamma(1-\phi) \mu_{W}^{0}+\phi\left(\mu_{\mathrm{A}}^{0}-k T \ln \gamma\right)
$$

where $\mu_{W}^{0}$ is the chemical potential of the water molecule in the absence of protein. $\mu_{A}^{0}$ is the chemical potential of the protein in the dilute protein limit. Here, $\gamma \equiv \Omega_{P} / \Omega_{W}$. The term $k T f(\phi)$ represents the entropy of mixing between the protein and the water molecules. ${ }^{7}$ The final term in eq 6 expresses the meanfield quadratic form of the net like-like and like-unlike interaction energies between the constituents of the binary solution. In fact, if we use a lattice model $^{7}$ to describe the energy of interaction between adjacent molecules in the solution, we can identify the parameter $\sigma E_{n e t}(A, W)$ as

$$
\sigma E_{n e t}(A, W)=\sigma\left[E_{A W}-\frac{\left(E_{A A}+E_{W W}\right)}{2}\right]
$$

Here $E_{A W}$ is the mean energy of a single protein-water lattice adjacency, $E_{A A}$ is the mean energy of a protein-protein adjacency, and $E_{W W}$ is the mean energy of a single water-water adjacency. The quantity $\sigma$ represents the total number of possible adjacencies associated with each protein.

The generalization of eq 5 appropriate for a ternary solution has the form

$$
\begin{array}{r}
g(\phi, \alpha, T)=\alpha g_{A}(\phi, T)+(1-\alpha) g_{B}(\phi, T)+k T \phi h(\alpha)+ \\
\alpha(1-\alpha) \phi^{2} \sigma E_{n e t}(A, B)
\end{array}
$$

In this equation $g_{B}(\phi, T)$ has the same structure as shown in eq 5 except that $A$ is to be replaced by $B$ with a corresponding change in eq 7 for $\sigma E_{\text {net }}(A, W)$ to give $\sigma E_{\text {net }}(B, W)$. The quantity

$$
h(\alpha)=\alpha \ln \alpha+(1-\alpha) \ln (1-\alpha)
$$

represents the ideal entropy of mixing among distinguishable proteins of type $A$ and $B$. The final term in eq 8 represents the contribution to the energy of the nonideal mixing of the two proteins, when the proteins each have the same volume and shape and when the mean-field approximation applies. A generalization to the ternary solution of the lattice model for the energy of interaction between constituent molecules gives, in perfect analogy to the expression for $\sigma E_{n e r}(A, W)$ and $\sigma E_{\text {ner }}(B, W)$, the expression

$$
\sigma E_{n e t}(A, B)=\sigma\left[E_{A B}-\frac{\left(E_{A A}+E_{B B}\right)}{2}\right]
$$

where again $\sigma$ is the total number of possible adjacencies associated with each protein in the solution. The lattice model clearly shows that the parameter $\sigma E_{n e t}(A, B)$ provides a measure of the difference in energy between the like-unlike $(A B)$ interprotein adjacencies and the average of the energies of the like-like $(A A$ or $B B)$ adjacencies. We may rewrite eq 8 in the following general form:

$$
g(\phi, \alpha, T)=k T\left\{U^{0}+U(\phi, \alpha, T)+\phi h(\alpha)\right\}
$$

In the mean-field picture

$$
k T U^{0}=\phi\left(\mu_{B}^{0}-k T \ln \gamma\right)+\gamma(1-\phi) \mu_{W}^{0}+\alpha \phi\left(\mu_{\mathrm{A}}^{0}-\mu_{B}^{0}\right)
$$

is the "standard" contribution to $g$ containing terms linear in $\phi$. As we shall see, these terms make no contribution to the location of the phase boundaries in the ternary solution. Also, using eq 8 we have

$$
\begin{aligned}
k T U(\phi, \alpha, T)= & k T f(\phi)-\phi^{2} \sigma\left\{\alpha E_{n e t}(A, W)+\right. \\
& \left.(1-\alpha) E_{n e t}(B, W)-\alpha(1-\alpha) E_{n e t}(A, B)\right\}
\end{aligned}
$$

This equation shows that the magnitudes of the parameters $E_{n e t}(A, B)$ and $E_{\text {net }}(A, W)-E_{\text {ner }}(B, W)$ determine the dependence of $U$ upon $\alpha$. As the protein becomes similar, both these parameters approach zero (see eq 10), and as would be expected on general grounds, $U$ becomes independent of $\alpha$.

Having presented the structure of the Gibbs free energy we may now consider how this enables the deduction of the compositions $\left(\alpha^{I}, \alpha^{I I}\right)$ and concentrations $\left(\phi^{I}, \phi^{I I}\right)$ in coexisting phases. If the temperature is adjusted appropriately for a given initial total protein volume fraction $\phi^{0}$ and composition $\alpha^{0}$, the solution will separate into two coexisting phases designated I and $\Pi$. The convenient variables which describe, at temperature $T$, such coexisting phases are $\left(\phi^{I}, \alpha^{I}\right),\left(\phi^{I I}, \alpha^{I I}\right)$, and $\left(V^{I} / V\right) \equiv x$ : five variables in all. The variable $x$ is the fraction of the total volume occupied by phase I. In principle, these five variables are determined for given initial $\phi^{0}, \alpha^{0}$ by the following five conditions. There are two conditions describing the conservation of the total number of proteins and the total number of proteins of type $A$ :

$$
\begin{gathered}
x \phi^{I}+(1-x) \phi^{I I}=\phi^{0} \\
x \alpha^{I} \phi^{I}+(1-x) \alpha^{I I} \phi^{I I}=\alpha^{0} \phi^{0}
\end{gathered}
$$

Also, there are three thermodynamic equilibrium conditions that require that the chemical potentials of the two proteins $\mu_{A} \equiv$ $\left(\partial G / \partial N_{A}\right)_{N_{B}, N_{W}, T}$ and $\mu_{B} \equiv\left(\partial G / \partial N_{B}\right)_{N_{A}, N_{W}, T}$ and the water chemical potential $\mu_{W} \equiv\left(\partial G / \partial N_{W}\right)_{N_{A}, N_{B}, T}$ each be equal in phase I and phase II. These chemical potentials can be expressed entirely in terms of $g(\phi, \alpha, T)$ according to

$$
\begin{aligned}
\mu_{A}(\phi, \alpha, T)=g(\phi, \alpha, T)+(1-\phi)(\partial g / \partial \phi)_{\alpha, T}+ \\
{[(1-\alpha) / \phi](\partial g / \partial \alpha)_{\phi, T} }
\end{aligned}
$$




$$
\begin{array}{r}
\mu_{B}(\phi, \alpha, T)=g(\phi, \alpha, T)+(1-\phi)(\partial g / \partial \phi)_{\alpha, T}- \\
{[\alpha / \phi](\partial g / \partial \alpha)_{\phi, T}} \\
\mu_{W}(\phi, \alpha, T)=(1 / \gamma) g(\phi, \alpha, T)-[\phi / \gamma](\partial g / \partial \phi)_{\alpha, T}
\end{array}
$$

Note also that, since $G=\Sigma N_{i} \mu_{i}$,

$$
g(\phi, \alpha, T)=\mu_{A} \alpha \phi+\mu_{B}(1-\alpha) \phi+\gamma \mu_{W}(1-\phi)
$$

It is convenient to construct symmetrized linear combinations of these three equations so as to cast the equilibrium conditions in a form which is a generalization to the ternary solution of the well-known "double tangency condition", 15 which determines the coexisting values $\phi^{I}$ and $\phi^{I I}$ in the case of binary mixtures from the $\phi$ dependence of $g(\phi, T)$ at each fixed temperature. For this purpose, we require that the linear combinations $\left(\mu_{A}-\mu_{B}\right),\left(\gamma \mu_{W}-\mu_{B}\right)$, and

$$
\begin{aligned}
\left\{\gamma \mu_{W}\left(1-\left(\phi^{I}+\phi^{I I}\right) / 2\right)+\right. & \mu_{A}\left(\alpha^{I} \phi^{I}+\alpha^{I I} \phi^{I I}\right) / 2+ \\
& \left.\mu_{B}\left(\left(1-\alpha^{I}\right) \phi^{I}+\left(1-\alpha^{I I}\right) \phi^{I I}\right) / 2\right\}
\end{aligned}
$$

must each have the same value in phase I and phase II. Mathematically these conditions are entirely equivalent to the condition that $\mu_{A}, \mu_{B}$, and $\mu_{W}$ each must have the same value in phase I and phase II. However, the equations for the individual chemical potentials, when expanded in a power series, in $\Delta \alpha$ $=\alpha^{I I}-\alpha^{I}$ and $\Delta \phi=\left(\phi^{l l}-\phi^{I}\right)$, are not independent, in lowest order, because of the Gibbs-Duhem relations. The third combination is chosen to overcome this difficulty. Note that this combination is $(g(I)+g(I I)) / 2$ for phase separation. Here $g(I) \equiv g\left(\phi^{I}, \alpha^{I}, T\right)$ and $g(I I) \equiv g\left(\phi^{I I}, \alpha^{I I}, T\right)$. If we use the definitions $\dot{\mathrm{g}}(\phi, \alpha, T) \equiv(\partial g(\phi, \alpha, T) / \partial \alpha)$ and $g^{\prime}(\phi, \alpha, T) \equiv$ $(\partial g(\phi, \alpha, T) / \partial \phi)$, the equilibrium conditions then give the following three equations:

$$
\begin{gathered}
\left(\dot{g}(I) / \phi^{I}\right)=\left(\dot{\mathrm{g}}(I I) / \phi^{I I}\right) \\
g^{\prime}(I)-\left(\alpha^{I} \dot{\mathrm{g}}(I) / \phi^{I}\right)=g^{\prime}(I I)-\left(\alpha^{I I} \dot{\mathrm{g}}(I I) / \phi^{I I}\right) \\
(g(I I)-g(I))+\left(\frac{g^{\prime}(I)+g^{\prime}(I)}{2}\right)\left(\phi^{I}-\phi^{I I}\right)+ \\
\left(\frac{\alpha^{I}-\alpha^{I I}}{2}\right)\left(\frac{\dot{\mathrm{g}}(I I) \phi^{I}}{\phi^{I I}}+\frac{\dot{\mathrm{g}}(I) \phi^{I I}}{\phi^{I}}\right)=0
\end{gathered}
$$

The five equations $(14 a, b)$ and $(17 a-c)$ determine, in principle, how each of the five variables $\left(\phi^{I}, \alpha^{I}\right),\left(\phi^{I I}, \alpha^{I I}\right)$, and $x$ depends on $T$, for given values of the overall starting volume fraction $\phi^{0}$ and composition $\alpha^{0}$. If we are only interested in points on the coexistence surface, then eqs $(14 a, b)$ are satisfied, and we can restrict our attention to the remaining eqs $(17 a-c)$. These comprise three relationships between four unknowns $\left(\phi^{I}, \alpha^{I}\right)$ and $\left(\phi^{I I}, \alpha^{I I}\right)$ at fixed temperature $T$. Thus, for example, if we choose $\phi^{I}$ as an independent variable, there can be a range of $\phi^{I}$ for which eqs $(17 a-c)$ predict corresponding values of $\alpha^{I}$ and $\left(\phi^{I I}, \alpha^{I I}\right)$. Therefore, by changing $\phi^{I}$ we generate a conjugate pair of curves in the $(\phi, \alpha)$ plane at fixed $T$. In our case these two curves form the descending and ascending limbs of a single binodal curve. By considering a sequence of such conjugate curves, constructed by using increments in the temperature $T$, we can sweep out the coexistence surface.

We now examine the mathematical solution of eqs $(17 a-c)$, when the form of $g(\phi, \alpha, T)$ is that given by eq 11. Insertion of this into eqs $(17 \mathrm{a}-\mathrm{c})$ shows that the term $k T U^{0}$, which is linear in $\phi$, completely cancels out of these three stability conditions.
Equation 17a takes the form

$$
\left(\frac{\dot{U}(I)}{\phi^{I}}-\frac{\dot{U}(I I)}{\phi^{I I}}\right)=\dot{h}(I I)-\dot{h}(I)
$$

Our further analysis of this problem is based on the view that the difference between our proteins is small and that the solution of the equilibrium conditions can be found in terms of an expansion in powers of the small composition difference $\Delta \alpha$ between coexisting phases. Here

$$
\Delta \alpha \equiv\left(\alpha^{I I}-\alpha^{I}\right)
$$

Indeed, as the proteins become more nearly identical to one another, $U$ becomes independent of $\alpha$ and hence $\dot{U} \rightarrow 0$. Thus $\dot{h}(I I) \rightarrow \dot{h}(I)$, and since $\dot{h}$ is a monotonic function of $\alpha$, it follows that $\boldsymbol{\alpha}^{I I} \rightarrow \boldsymbol{\alpha}^{I}$ : the compositions of both phases become identical. In our expansion method we regard $\Delta \alpha$ as being small compared to unity. Under these conditions we may expand the right-hand side of eq 18 in powers of $\Delta \alpha$ about $\bar{\alpha} \equiv\left(\alpha^{I}+\alpha^{I I}\right) / 2$, and find that $\dot{h}(I I)-\dot{h}(I)=(\Delta \alpha / \bar{\alpha}(1-\bar{\alpha}))+O(\Delta \alpha)^{3}$. We therefore observe that $\Delta \alpha$ is of the order of $\dot{U}$. Using the mean-field expression for $U(\phi, \alpha, T)$ given in eq 13 we may calculate the left-hand side of eq 18 and find, on retaining terms linear in $\Delta \alpha$, the following relationship between $\Delta \alpha$ and $\left(\phi^{I I}-\phi^{I}\right)$ :

$$
\begin{array}{r}
\Delta \alpha=\left(\phi^{I I}-\phi^{I}\right) \bar{\alpha}(1-\bar{\alpha}) \frac{\sigma}{k T}\left\{\left(E_{n e t}(A, W)-E_{n e t}(B, W)\right)-\right. \\
\left.(1-2 \bar{\alpha}) E_{n e t}(A, B)\right\}
\end{array}
$$

If we remember that the binodal curve represents, at fixed temperature $T$, the locus of pairs of coexisting values of $\left(\phi^{\prime l}, \alpha^{I l}\right)$ and $\left(\phi^{I}, \alpha^{I}\right)$, then eq 20 shows how the slope, $\Delta \alpha /\left(\phi^{I I}-\phi^{I}\right)$, of the "tie lines" connecting coexisting phases on the binodal curve should vary with the mean composition $\bar{\alpha}$. Equation 20 also confirms quantitatively that $\Delta \alpha$ is directly proportional to the parameters $\left(E_{\text {net }}(A, W)-E_{n e t}(B, W)\right)$ and $E_{n e t}(A, B)$, which characterize the difference between proteins. From a practical point of view, however, eq 20 does not provide an accurate means of determining those parameters from our experimental data, because of experimental uncertainties in the values of $\left(\phi^{I I}, \alpha^{I I}\right)$ and $\left(\phi^{I}, \alpha^{I}\right)$ and the fact that $\bar{\alpha}(1-\bar{\alpha}) \rightarrow 0$ for $\bar{\alpha}=0$, $\bar{\alpha}=1$, and $(1-2 \bar{\alpha}) \rightarrow 0$ at $\bar{\alpha}=1 / 2$.

Turning now to the second stability condition eq $17 \mathrm{~b}$, we find the following relationship between $U^{\prime}(I)$ and $U^{\prime}(I I)$ :

$$
\left(U^{\prime}(I)-U^{\prime}(I I)\right)=-\left(\frac{\Delta \alpha}{2}\right)\left(\frac{\dot{U}(I)}{\phi^{I}}+\frac{\dot{U}(I I)}{\phi^{I I}}\right)+O(\Delta \alpha)^{3}
$$

Furthermore, using the fact that $\dot{U} \sim \Delta \alpha$, it follows at once that the first term on the right-hand side of eq 21 in lowest order is quadratic in $\Delta \alpha$. Thus, if we neglect terms quadratic in $\Delta \alpha$, the equilibrium condition expressed in eq $17 \mathrm{~b}$ or eq 21 implies that

$$
U^{\prime}\left(\phi^{I}, \alpha^{I}, T\right) \cong U^{\prime}\left(\phi^{I l}, \alpha^{I l}, T\right)
$$

If, furthermore, one expands these functions around any value of $\alpha$ between $\alpha^{I}$ and $\alpha^{I I}$, one finds on neglecting terms quadratic in $\Delta \alpha \equiv\left(\alpha^{I I}-\alpha^{\prime}\right)$ that

$$
U^{\prime}\left(\phi^{I}, \alpha, T\right) \approx U^{\prime}\left(\phi^{I I}, \alpha, T\right)
$$

Thus, at $\phi^{I}$ and $\phi^{I I}$, the tangents $(\partial U / \partial \phi)_{\phi^{I}, \alpha}$ and $(\partial U / \partial \phi)_{\phi^{\mathbb{I}}, \alpha}$ are essentially equal to one another.

We now turn to eq $17 \mathrm{c}$ and insert eq 11 . If we neglect all terms quadratic in $\Delta \alpha$, we find 


$$
(U(I I)-U(I)) \simeq\left(\phi^{I I}-\phi^{I}\right)\left(\frac{U^{\prime}(I)+U^{\prime}(I I)}{2}\right)
$$

If we now note that $U(I I) \equiv U\left(\phi^{I I}, \alpha^{I I}, T\right)$ and $U(I) \equiv U\left(\phi^{I}, \alpha^{I}, T\right)$, and once again express $\alpha^{I}$ and $\alpha^{I I}$ in terms of $\Delta \alpha$ and $\alpha$, where $\alpha^{I}<\alpha<\alpha^{I I}$, then one can expand $U$ and $U^{\prime}$ about $\alpha$ to show that, upon neglecting terms of order $(\Delta \alpha)^{2}$ and higher, eq 23a can be written as

$$
\begin{aligned}
& \left(U\left(\phi^{I I}, \alpha, T\right)-U\left(\phi^{I}, \alpha, T\right)\right)= \\
& \left(\phi^{I I}-\phi^{I}\right)\left(\frac{U^{\prime}\left(\phi^{I I}, \alpha, T\right)+U^{\prime}\left(\phi^{I}, \alpha, T\right)}{2}\right)
\end{aligned}
$$

Thus, eqs $22 \mathrm{~b}$ and $23 \mathrm{~b}$ show that the values of $\phi^{I I}$ and $\phi^{I}$ are determined, for small $\Delta \alpha$, by a double-tangency condition having precisely the same form as that which applies in the binary mixture case. ${ }^{7,15}$ Rewriting now the mean-field expression for $U(\phi, \alpha, T)$ (eq 13) as

$$
U(\phi, \alpha, T)=f(\phi)-\phi^{2}\left(\frac{\sigma E_{n e t}(\alpha, T)}{k T}\right)
$$

we observe that the entire dependence on $\alpha$ and $T$ is contained in the single parameter $\left(\sigma E_{n e t}(\alpha, T) / k T\right)$ where

$$
\begin{array}{r}
E_{n e t}(\alpha, T)=\alpha E_{n e t}(A, W, T)+(1-\alpha) E_{n e t}(B, W, T)- \\
\alpha(1-\alpha) E_{n e t}(A, B, T)
\end{array}
$$

It is instructive to consider the implementation of the doubletangency condition. Indeed, such a consideration leads us to a general equation for the coexistence surface. For eqs $22 \mathrm{~b}$ and $23 \mathrm{~b}$ to have a solution different from $\phi^{I}=\phi^{I I}$ the parameter ( $\left.\sigma E_{\text {ned }} / k T\right)$ must be greater than some critical value $\left(\sigma E_{\text {ned }} / k T\right)_{\text {crit }}$. This critical value corresponds to the condition in which $\phi^{I} \rightarrow$ $\phi^{I I} \rightarrow \phi_{c}$, where $\phi_{c}$ is the critical protein volume fraction. We have shown ${ }^{7}$ in a mean-field approximation that in this limit $\left(\sigma E_{\text {ned }} k T\right)_{\text {crit }}=f^{\prime}\left(\phi_{c}\right) / 2=13.1$. Thus, all values of $\left(\sigma E_{\text {net }} / k T\right)$ corresponding to two coexisting phases can be expressed in terms of a new parameter $t$ in accordance with the definition $\left(\sigma E_{n e t} / k T\right)=(13.1 / t)$ where $0<t \leq 1$. Furthermore, by applying the conditions (22b) and (23b) the corresponding values of $\phi^{I}$ and $\phi^{I I}$ can be found. Thus, for each $t(0<t \leq 1)$, these equations provide two values of $\phi$. Indeed the function $t(\phi)$, which we shall denote as "the normalized scaling section" and which has the same value for both $\phi=\phi^{I}$ and $\phi=\phi^{l l}$, can be used to provide an expression for the coexistence surface, valid in the domain of small $\Delta \alpha$. According to our analysis above, for given $\phi$ and $\alpha$ the corresponding temperature $\left(T_{p h}(\phi, \alpha)\right)$ satisfies the equation:

$$
\frac{\sigma E_{n e t}\left(\alpha, T_{p h}(\phi, \alpha)\right)}{k T_{p h}(\phi, \alpha)}=\frac{13.1}{t(\phi)}
$$

This is the equation of the coexistence surface $T_{p h}(\phi, \alpha)$. Observe that this equation has the property that, for fixed $\alpha$, the $\left(T_{p h}(\phi)\right)_{\alpha}$ sections of the coexistence surfaces have shapes determined essentially by the normalized scaling section $t(\phi)$. Furthermore, eqs 25 and 26 show that as $\alpha$ changes, these sections simply are displaced vertically, in harmony with the experimental observations. Similarly for fixed $\phi$ the curves of $\left(T_{p h}(\alpha)\right)_{\phi}$ have the quadratic form determined by eqs 26 and 25 . Finally the binodal curve is the locus of $(\phi, \alpha)$ points which satisfies eq 26 for $T_{p h}(\phi, \alpha)=$ constant.

\section{Comparison between the Theory and the Experimental Results}

We now obtain the three fundamental energy parameters: $\sigma E_{\text {ner }}(A, W), \sigma E_{\text {ner }}(B, W)$, and $\sigma E_{\text {ner }}(A, B)$ appearing in the normal-

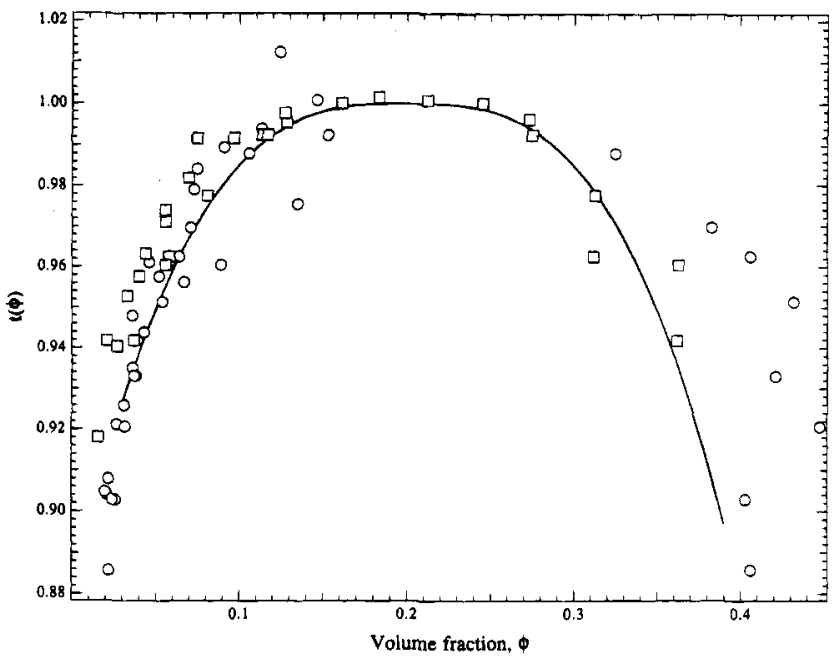

Figure 4. Deduction of the normalized scaling section $t(\phi)$ using the experimental data for $\alpha=0(0)$ and $\alpha=1.0(\square)$. The solid line is an empirical fit to the data over the domain $0.03 \leq \phi \leq 0.39$ and has the form $t(\phi)=\left(1-15\left|\phi-\phi_{c}\right|^{3}\right)$.

ized Gibbs free energy and examine the extent to which these parameters, when used in the theory, can describe the full range of our experimental findings.

We have, in fact, previously determined the magnitude and temperature dependence of the parameters $\sigma E_{n e t}(A, W, T)$ and $\sigma E_{\text {net }}(B, W, T)$ from measurements of the equation of state and coexistence curves of aqueous solutions of pure $A$ and pure $B$ proteins. This analysis shows that the temperature dependence of these parameters is essentially linear and that, for $T=T_{c}(A)$ or $T=T_{c}(B)$, which are the critical temperatures of pure $\mathrm{A}$ or B solutions, $\sigma E_{\text {net }}\left(A, W, T_{c}(A)\right)=13.1 k T_{c}(A)$ and $\sigma E_{\text {net }}\left(B, W, T_{c}(B)\right)$ $13.1 k T_{c}(B)$. Also, we have found ${ }^{7}$ that $\partial\left(\sigma E_{n e t}(A, W) / \partial(k T)\right)=$ -13 and $\partial\left(\sigma E_{n e t}(B, W) / \partial(k T)\right)=-9$. Thus two of the three parameters of the theory are in fact fixed by prior determination, using binary solution data. We can deduce the third parameter of the theory $\sigma E_{\text {ner }}(A, B)$ as follows. First note that eq 26 provides a means to determine $t(\phi)$ by using the experimental measurements of $T_{p h}(\phi, \alpha)$ for $\alpha=0$ and $\alpha=1$ and the previous determinations of $\sigma E_{\text {ner }}(A, W, T)$ and $\sigma E_{\text {net }}(B, W, T)$. This is shown in Figure 4, which demonstrates that within experimental error $t$ is indeed a function only of $\phi$. In Figure 4 we show as a solid line an empirical form for $t(\phi)$, which, within experimental error, represents the data for $t(\phi)$ in the domain $0.03 \leq \phi \leq$ 0.39 .

Equation 26 may now be used to deduce the parameter $\sigma E_{\text {ner }}(A, B, T)$ from the form of the sections corresponding to fixed values, $\phi_{\lambda}$, of $\phi$. Such sections, as shown in Figure 2, according to our theory follow a basically quadratic dependence on $\alpha$ in accordance with the equation

$$
\begin{aligned}
T_{p h}\left(\phi_{\lambda}, \alpha\right)=\frac{t\left(\phi_{\lambda}\right)}{13.1} \frac{\sigma}{k}\left\{\alpha E_{n e t}\left(A, W, T_{p h}\right)+\right. \\
\left.\quad(1-\alpha) E_{n e t}\left(B, W, T_{p h}\right)-\alpha(1-\alpha) E_{n e t}(A, B)\right\}
\end{aligned}
$$

It follows that each of the curves in Figure 2 can be used in conjunction with the known temperature dependence of $\sigma E_{\text {net }}(A, W, T)$ and $\sigma E_{\text {ner }}(B, W, T)$ to fix the numerical value of $\sigma E_{\text {ner }}(A, B, T)$. By so doing we have determined that $\sigma E_{\text {ner }}(A, B, T) /$ $k T_{c}(A)=-1.0 \pm 0.05$, independent of the temperature. Our theoretical analysis has shown that the dimensionless parameters which quantitatively describe the "difference" between the proteins are essentially $\epsilon_{1} \equiv\left[\sigma E_{n e t}(A, W)-\sigma E_{\text {ner }}(B, W)\right] / k T_{c}(A)$ and $\epsilon_{2} \equiv \sigma E_{n e r}(A, B) / k T_{c}(A)$. According to our findings these two quantities are respectively $\sim 2.2$ and $\sim-1.0$. Thus both 
these dimensionless quantities are comparable in size. In the context of the lattice model for the interparticle interaction energies, the negative sign for $E_{\text {net }}(A, B)$ implies that, in the present case, the net attractive (negative) like-unlike bond energy $E_{A B}$ is in fact numerically larger than the mean attractive like-like bond energy $\left(\left(E_{A A}+E_{B B}\right) / 2\right)$. In Figure 2 we also show as lines the theoretical prediction for the $\left(T_{p h}(\alpha)\right)_{\phi}$ sections. To obtain these curves we have used the magnitudes of $\sigma E_{n e t}(A, W, T)$ and $\sigma E_{\text {net }}(B, W, T)$ as obtained from $T_{c}(A)=311.6$ $\mathrm{K}$ and $T_{c}(B)=279.6 \mathrm{~K}$. These are the appropriate critical temperatures for the data presented in the figure (see discussion below). Also we have used the linear temperature dependence as given above for the energy parameters. In view of the uncertainties in the experimental deductions of $t(\phi)$ and the energy parameters, the theoretical predictions are in satisfactory agreement with the data.

Having established the form of $t(\phi)$ and the magnitude and temperature dependence of each of the energy parameters, we are now in a position to provide theoretical predictions for all the remaining experimental data.

We examine first the constant $\alpha$ sections $\left(T_{p h}(\phi)\right)_{\alpha}$. In Figure 1 the dashed lines represent the theoretical predictions. Here we used the values $T_{c}(A)=309.5 \mathrm{~K}$ for $\alpha=1$ and $T_{c}(B)=$ $278.5 \mathrm{~K}$ for the $\alpha=0$ sections, as given in ref 1 . The remaining data in Figure 1 were obtained under slightly different preparative conditions, for which we found $T_{c}(A)=311.6 \mathrm{~K}$ and $T_{c}(B)$ $=279.6 \mathrm{~K}$. These latter conditions also apply to the data shown in Figures 2 and 3 . The theoretical predictions appear to satisfactorily reproduce the characteristic vertical displacement of the $\left(T_{p h}(\phi)\right)_{\alpha}$ sections, found experimentally, as the composition $\alpha$ is increased from 0 to 1 . The theory also predicts the vertical displacements found experimentally for the $\left(T_{p h}(\alpha)\right)_{\phi}$ sections as shown in Figure 2.

We examine next the data shown in Figure 3, which represents coexisting values of $\phi$ and $\alpha$ at $T=20^{\circ} \mathrm{C}$. In this figure, the solid curve is the binodal curve as computed from eq 26. The solid straight lines connect the theoretically calculated points $\left(\phi^{I}, \alpha^{I}\right)$ and $\left(\phi^{I I}, \alpha^{I I}\right)$ shown the represent the so-called "tie lines" appropriate to each of the values of $\phi^{0}$ and $\alpha^{0}$ presented in Table 1 . The coexisting values of $\phi$ and $\alpha$ were determined by using the following line of reasoning. The conservation equations, eqs $14 \mathrm{a}$ and $14 \mathrm{~b}$, in effect relate $\left(\phi^{I}, \alpha^{\prime}\right)$ and $\left(\phi^{I I}, \alpha^{I I}\right)$ to $\left(\phi^{0}, \alpha^{0}\right)$. Furthermore, the binodal curve, $(\phi(\alpha))_{T}$, provides a relation between both $\alpha^{I}$ and $\phi^{I}$ and $\alpha^{I l}$ and $\phi^{I I}$. Thus, we now have a relationship between $\alpha^{I}, \alpha^{I I}$ and $\phi^{0}, \alpha^{0}$. Another such relationship between $\alpha^{I}$ and $\alpha^{I I}$ is contained in eq 20 for the slope of the tie line. Solving these two equations for $\left(\alpha^{I}, \alpha^{I I}\right)$ we obtain at once the corresponding values of $\left(\phi^{I}, \phi^{I I}\right)$ from the binodal curve. Hence we find the coexisting values of $\phi$ and $\alpha$ corresponding to given $\phi^{0}, \alpha^{0}$ at fixed temperature $T$. The prediction shown in Figure 3 must be regarded as satisfactory considering the uncertainties in the experimental determination of the composition and volume fraction in the coexisting phases, particularly in view of the long time needed to reach phase equilibrium.

\section{Summary and Conclusions}

We have presented experimental findings which describe the separation of ternary aqueous solutions of the bovine lens proteins $\gamma_{\mathrm{ma}}$ (protein A) and $\gamma_{\mathrm{mb}}$ (protein B) into coexisting liquid phases. The data include segments of the coexistence surface corresponding to constant composition $(\alpha)$ sections $\left(T_{p h}(\phi)\right)_{\alpha}$, constant volume fraction $(\phi)$ sections $\left(T_{p h}(\alpha)\right)_{\phi}$, and coexisting values $\left(\phi^{I}, \alpha^{I}\right)$ and $\left(\phi^{I I}, \alpha^{I I}\right)$ of protein volume fraction and composition which define the form of the binodal curve at fixed temperature $T=20^{\circ} \mathrm{C}$. To understand the underlying physical and chemical factors which produce this phase separation, we have constructed a mean-field theory using a normalized Gibbs free energy $g(\phi, \alpha, T)$, which is a natural extension of that found appropriate for binary aqueous-protein solutions. The normalized Gibbs free energy $g(\phi, \alpha, T)$ contains three energy parameters $\sigma E_{\text {net }}(A, W, T), \sigma E_{\text {ner }}(B, W, T)$, and $\sigma E_{\text {net }}(A, B, T)$. The physical significance of these energy parameters is identified, using a lattice model for the ternary solution, in terms of the mean values of the individual protein-water, water-water, and protein-protein bond interaction energies. Using this $g(\phi, \alpha, T)$ one can obtain the basic chemical potentials $\mu_{\mathrm{A}}(\phi, \alpha, T), \mu_{B}(\phi, \alpha, T)$, and $\mu_{W}(\phi, \alpha, T)$ of each protein species and the water. The thermodynamic condition that each of these three chemical potentials be identical in the two coexisting phases (I,II) determines in principle the coexisting values of $\left(\phi^{I}, \alpha^{I}\right)$ and $\left(\phi^{I I}, \alpha^{I I}\right)$. We have explicitly implemented these conditions of thermodynamic equilibrium using a power series expansion procedure appropriate when the proteins, in effect, are slightly dissimilar, as is the case for our system. Using this method of analysis, we have obtained equations for the coexistence surface and can determine analytically coexisting values of $\left(\phi^{I}, \alpha^{I}\right)$ and $\left(\phi^{I}, \alpha^{I}\right)$ along the binodal curve at fixed temperature $T$. Detailed comparison between the theory and experimental results requires in fact the determination of just one of the energy parameters $\sigma E_{\text {ner }}(A, B, T)$ since the other two are known from independent measurements on binary aqueous solutions of pure $\gamma_{\mathrm{Illa}}$ and $\gamma_{\mathrm{IIb}}$. Using a very limited subset of the data, we have determined that $\sigma E_{n e r}(A, B, T)$ is a constant independent of the temperature and equal to $(-1.0 \pm 0.05) k T_{c}(A)$. Having fixed this constant, it is possible to satisfactorily predict all the experimental measurements including the $\left(T_{p h}(\phi)\right)_{\alpha}$ and $\left(T_{p h}(\alpha)\right)_{\phi}$ sections of the coexistence surface and the values of $\left(\phi^{I}, \alpha^{I}\right)$ and $\left(\phi^{I I}, \alpha^{I I}\right)$ along the binodal curve at fixed temperature $T$. Of course, this theory can also be used to calculate thermodynamic properties of the solution phase other than those measured in this paper. Such properties include the equation of state, i.e., the osmotic pressure $\pi(\phi, \alpha, T)$, and the heat capacity, at any point in the phase diagram.

The success of the present mean-field theory indicates that we now have a promising theoretical basis for understanding the entire range of equilibrium thermodynamic properties and phase boundaries of ternary protein solutions, for the case of proteins which are not too dissimilar. We see that the observable equilibrium properties are determined by three energy parameters $\sigma E_{\text {net }}(A, W, T), \sigma E_{\text {ner }}(B, W, T)$, and $\sigma E_{\text {net }}(A, B, T)$ and by the form of the entropy of mixing between the proteins and the aqueous medium, as represented by $f(\phi)$. The present theory provides means by which these parameters can be deduced from a limited number of measurements in experimentally convenient regions of the phase diagram and then used to reliably predict the solution properties in other less accessible regions.

We also keep in mind that the physical significance of each of these energy parameters is clearly delineated in terms of bond energies by the lattice model. Thus, since the effect of changes on each of these parameters produces clearly deducible consequences for the location of the coexistence surface, it now becomes possible to establish rational design and testing methods for the discovery of protein or solvent modifying agents which selectively alter the bond interaction energies so as to relocate the coexistence surface into desired positions in the phase diagram. In this way, for example, one should be able to maintain the solubility of multicomponent protein-water solutions in the living cell by chemical means, thereby counteracting posttranslational modifications which otherwise 
would lead to protein condensation, such as occurs in the case of cataract disease. Finally, we must recognize that it is necessary to extend the implementation of this theory to the important case of ternary solutions in which the proteins are quite different in size and in the magnitude of their respective bond interaction energies.

Acknowledgment. This research was supported in part by the National Science Foundation/SGER under Grant 9222712DMR, by the National Institutes for Health under Grant R01 EYO5127, and by the National Science Foundation Materials Research Laboratory under Grant DMR 90-22933.

\section{References and Notes}

(1) Broide, M. L.; Berland, C. R.; Pande, J.; Ogun, O. O.; Benedek, G. B. Proc. Natl. Acad. Sci. U.SA. 1991, 88, 5660.

(2) Benedek, G. The Molecular Basis of Cataract Formation. In Human Cataract Formation; CIBA Foundation Symposium 106; Pitman Publication Ltd.: London, 1984; $\mathrm{p} 237$.

(3) Eaton, W. A.; Hofrichter, J. In Advances in Protein Chemistry; Anfinsen, C. B., Edsall, J. T., Richards, F. M., Eisenberg, D. S., Eds.; Academic Press: San Diego, 1990; Vol. 40, p 63.
(4) Glenner, G. G. The New England Journal of Medicine; 1980, 302, Part I, 1283 and Part II, 1333.

(5) Crowther, R. A. Biochem. Biophys. Acta 1991, 1096, 1. 573.

(6) Hay, R.; Andley, U. P.; Petrash, J. M. Exp. Eye Res. 1994, 58,

(7) Fine, B. M. Ph.D Thesis, M.I.T., 1994. Thurston, G. M.; Blankschtein, D.; Benedek, G. B., manuscript in preparation.

(8) Thomson, J. A.; Schurtenberger, P.; Thurston, G. M.; Benedek, G. B. Proc. Natl. Acad. Sci. U.S.A. 1987, 84, 7079.

(9) Bjork, I. Exp. Eye Res. 1964, 3, 254.

(10) Siezen, R. J.; Kaplan, E. D.; Anello, R. D. Biochem. Biophys. Res. Commun. 1985, 127, 153.

(11) McDermott, M. J.; Gawinowicz-Kolks, M. A.; Chiesa, R.; Spector, A. Arch. Biochem. Biophys. 1988, 262, 609.

(12) Berland, C. R.; Broide, M. L.; Pande, J.; Ogun, O.; Benedek, G. B. Proc. Natl. Acad. Sci. U.S.A. 1992, 89, 1214.

(13) van Holde, K. E. Physical Biochemistry; Prentice-Hall: Englewood Cliffs, NJ; 1985; Chapter 2.

(14) Schurtenberger, P.; Chamberlin, R. A.; Thurston, G. M.; Thomson, J. A.; Benedek, G. B. Phys. Rev. Lett. 1989, 63, 2064.

(15) Prigogine, I.; Defay, R. Chemical Thermodynamics, 3rd ed.; Longmans: London, 1965; Chapter 16.

JP941906S 